\title{
Integrating Spectroscopy with Potato Disease Management
}

J. J. Couture ${ }^{\dagger}$ and A. Singh, Department of Forest and Wildlife Ecology, A. O. Charkowski, Department of Plant Pathology, and R. L. Groves, Department of Entomology, University of Wisconsin-Madison, Madison 53706; S. M. Gray, Emerging Pest and Pathogen Research Unit, United States Department of Agriculture Agricultural Research Service (USDA-ARS), and Section of Plant Pathology and Plant-Microbe Biology, Cornell University, Ithaca, NY 14850; P. C. Bethke, Vegetable Crops Research Unit, USDA-ARS, and Department of Horticulture, University of Wisconsin-Madison; and P. A. Townsend, Department of Forest and Wildlife Ecology, University of Wisconsin-Madison

\begin{abstract}
Spectral phenotyping is an efficient method for the nondestructive characterization of plant biochemical and physiological status. We examined the ability of a full range (350 to $2,500 \mathrm{~nm}$ ) of foliar spectral data to (i) detect Potato virus $Y$ (PVY) and physiological effects of the disease in visually asymptomatic leaves, (ii) classify different strains of PVY, and (iii) identify specific potato cultivars. Across cultivars, foliar spectral profiles of PVY-infected leaves were statistically different $(F=96.1, P \leq$ 0.001 ) from noninfected leaves. Partial least-squares discriminate analysis (PLS-DA) accurately classified leaves as PVY infected (validation $\kappa=$ 0.73 ) and the shortwave infrared spectral regions displayed the strongest correlations with infection status. Although spectral profiles of different
\end{abstract}

A growing global population has increased demands on agricultural production systems, placing pressure on growers to implement precision management practices. Increasing yield depends on minimizing disease and optimally managing inputs such as fertilizer and pesticides while accounting for changes in the environment and regulatory concerns over water availability and quality. One way this might be accomplished is use of advanced monitoring techniques to aid in precision crop management. Currently, the most widely used approach for monitoring stress and disease in plants is manual scouting, which can be both time consuming and subjective (Sankaran et al. 2010). Aerial imaging using multispectral cameras that generate a normalized difference vegetation index or similar indices are in use by some producers to scout fields for problem areas but the data collected cannot be used to unambiguously identify an underlying cause. Molecular techniques are being developed to identify specific viral strains and uncover plant resistance strategies (Di Carli et al. 2012) but these approaches require sample processing

Present address of J. J. Couture: Departments of Entomology and Forestry and Natural Resources and Center for Plant Biology, Purdue University, 901 W. State St., West Lafayette, IN 47907.

Present address of A. Singh: Department of Agricultural and Biological Engineering. University of Florida, 1741 Museum Rd., Gainesville 32611.

Present address of A. O. Charkowski: Department of Bioagricultural Sciences and Pest Management, Colorado State University, 307 University Ave., Fort Collins 80523

${ }^{\dagger}$ Corresponding author: J. J. Couture; E-mail: couture@purdue.edu

Funding: This work was funded, in part, by a USDA Specialty Crops Research Initiative grant (2014-51181-22373) to Cornell University, with subaward 73999-10420 to the University of Wisconsin-Madison. Additional support was provided by the Wisconsin Potato Industry Board and Wisconsin Potato and Vegetable Growers Association, as well as USDA Hatch awards WIS01874 and IND011490 and Purdue Center for Plant Biology.

Accepted for publication 25 April 2018.

@ 2018 The American Phytopathological Society procedures that are not easily or rapidly deployed at the scale of agricultural field production.

Reflectance spectroscopy has emerged as a promising tool for agricultural management (Martinelli et al. 2015; Sankaran et al. 2010) because it is a nondestructive, rapid, and relatively low-cost approach to monitoring vegetation status. Reflectance spectroscopy of vegetation relies on the interaction of light with plant chemical and structural composition and water content (Curran 1989). Reflection of light in the visible (400 to $700 \mathrm{~nm}$ ), near-infrared (NIR, 700 to $1,100 \mathrm{~nm}$ ), and short-wave infrared (SWIR, 1,100 to 2,400 nm) can provide a comprehensive assessment of shifts in visual symptoms (e.g., pigments and leaf color) and the underlying biochemical (e.g., nutrient composition and secondary metabolism) and physiological (e.g., photosynthetic activity and water relations) responses to disease or stress (Arens et al. 2016; Cotrozzi et al. 2017; Couture et al. 2013, 2015; Romero et al. 2017). Applications of spectroscopy for use in agriculture have largely focused on disease detection (Arens et al. 2016; Mahlein et al. 2012; Naidu et al. 2009), classification strategies (Rumpf et al. 2010), development of narrow- or broad-band disease indices (Mahlein et al. 2013), and relating vegetation indices sensitive to plant stress to plant disease status (Huang et al. 2007; Romero et al. 2017). Hyperspectral data are also being used to identify or classify plant cultivars and genotypes ( $\mathrm{Li}$ and He 2008; Li et al. 2007). In addition, spectroscopy has the potential to predict plant biochemical constituents such as nutrients and secondary metabolites (Asner and Martin 2008; Asner et al. 2011; Couture et al. 2013, 2016; Kleinebecker et al. 2009; Petisco et al. 2006; Serbin et al. 2014) and plant physiological status or specific physiological processes (Cotrozzi et al. 2017; Gamon et al. 1992, 1995; Serbin et al. 2012) based on variations in the optical properties of foliage. The use of hyperspectral data to detect disease in crops has been explored less and represents a promising and novel approach for the classification of disease status and strain identification as well as for nondestructive quantification of specific biochemical and physiological responses of plants to disease.

Potato (Solanum tuberosum L.) is an important food crop worldwide, ranking fourth in production behind wheat, rice, and maize. Unlike wheat, rice, and maize, potato is vegetatively propagated, with tubers serving as the planting material for the vast majority of potato crops worldwide. Vascular pathogens that infect the mother plant are transmitted to most or all progeny tubers (seed tubers), 
limiting the yield potential of all subsequent plants derived from an infected plant (seed lot degeneration) (Frost et al. 2013). Viruses are the most significant cause of seed lot degeneration, with the aphid-transmitted Potyvirus Potato virus Y (PVY) being the most important potato virus worldwide. In North America, PVY is the most common reason for rejection of seed lots from certification and one of the most common potato diseases. The foliar symptoms of PVY include mosaic, leaf drop, and interveinal necrosis, and this virus can also cause necrotic rings on potato tubers (Gray et al. 2010). Unfortunately, detection based on foliar symptoms is challenging because several widely grown North American cultivars exhibit transient or mild foliar symptoms with all PVY strains. In addition, the strains of PVY that have become prevalent during the past two decades in general induce milder foliar symptoms on most potato cultivars than on the cultivars they are replacing. Therefore, the historical method of identifying seed lots with high PVY incidence via visual inspection is less effective (Frost et al. 2013).

Seed potato certification agencies certify seed potato crops for both disease and cultivar purity, and a second common reason for rejection of seed lots from certification is cultivar mixture (Frost et al. 2013; Guenthner et al. 2012). Cultivar identity is currently validated through grower records and visual inspection. Visual identification of cultivars requires considerable expertise and has become more difficult in recent years due to an increase in the number of potato cultivars grown in North America. DNA analyses can be used to differentiate cultivars (Coombs et al. 2004; Douches and Ludlam 1991) but there are no high-throughput methods for cultivar identification.

The goal of this study was to determine whether hyperspectral data are a potential tool for potato management. Specifically, we sought to (i) detect the presence of PVY in asymptomatic leaves, (ii) classify different strains of PVY in living foliar tissue, (iii) identify specific potato cultivars, and (iv) quantify the effects of PVY on potato plant biochemistry and physiology.

\section{Materials and Methods}

Two sets of field measurements were used to assess the utility of hyperspectral data in potato disease management. First, to test the ability of spectral data to detect the presence of PVY in asymptomatic leaves and identify specific potato cultivars, foliar spectral measurements were collected, leaves were tested for presence of PVY, and specific PVY strains were identified on numerous potato cultivars at the Post-Harvest Test (PHT) in Waialua, Oahu, HI $(21.5712 \mathrm{~N}$, $-158.1518 \mathrm{~W})$. Second, to quantify specific biochemical, physiological, and morphological changes occurring in plants infected with PVY, foliar spectral measurements and leaf temperature were collected, regardless of the presence of visual symptoms, on PVYinfected and non-PVY-infected potato plants at the University of Wisconsin Hancock Agricultural Research Station (HARS), Hancock, WI (44.1205 N, $-89.5336 \mathrm{~W})$.

Field descriptions. 2015 PHT experiments. Spectral measurements were collected on 5 to 9 January 2015 at the PHT for PVY detection and cultivars discrimination. Seed potato samples, which consisted of whole, B-size tubers, were planted in early December 2014 in two adjacent rows of 200 tubers/row for each lot, and standard agronomic management practices were used to manage the integrity of the developing plant canopy for visual inspections. Potato plants were in the preflowering stage at time of spectral measurements.

2014 Mechanical inoculation experiments. Spectral measurements were collected from four commercially important cultivars (Atlantic, Russet Norkotah, Dark Red Norland, and Superior) to quantify the effect of PVY on potato foliar chemistry, morphology, and physiology. Individual plants were established from either tissue culture or certified seed tubers in the Walnut Street Greenhouse at the University of Wisconsin-Madison. Each seed tuber or plantlet was grown in Metro Mix potting media in a $20-\mathrm{cm}$ pot with a photoperiod of $16 \mathrm{~h}$ of light and $8 \mathrm{~h}$ of darkness for plant growth; then, light cycles were changed to 12 of light and $12 \mathrm{~h}$ of darkness to encourage tuber initiation. The inoculum used for this study was a PVYNTN (strain NY090029) obtained from Cornell University (Ithaca, NY).
Inoculum was prepared from infected tobacco leaf tissue ground in $0.01 \mathrm{M}$ potassium phosphate buffer $(\mathrm{pH} 7.4)$ at a ratio of approximately 1:10 (tissue/liquid [wt/wt]). Three young leaves in the upper one-third of the plant canopy were selected for mechanical sap inoculation using $\mathrm{CO}_{2}$-pressurized artist air brushes under modest pressure (50 to $60 \mathrm{psi}$ ). Inoculations were repeated three times during the preflower stage of crop development to increase the likelihood of infection. Harvested tubers were stored until they lost dormancy and then planted at the University of Wisconsin HARS, Hancock, in 2014. Stored tubers were planted in a randomized complete block experimental design with eight treatment levels (four cultivars by two levels of infection per cultivar), and treatments were randomized within each of four experimental blocks. Each replicate consisted of a single $6.5-\mathrm{m}$ row containing 20 plants/row within each block spaced $1 \mathrm{~m}$ apart, and blocks were separated by 3-m unplanted allies. Potato plants were grown using standard agronomic practices for irrigated potato in Wisconsin. Plants were measured 16 August 2014 and were in the tuberization stage at the time of spectral measurements.

Foliar spectral measurements. Fresh-leaf reflectance was measured using an SVC 1024i high-resolution full-range (350 to 2,500 nm) spectroradiometer (Spectral Vista Corporation, New York). All spectral measurements were collected from the leaf adaxial surface using a leaf-clip assembly attached to a plant probe with an internal, standardized, halogen light source. Reflectance was measured once on each leaf, with each data point being an average of multiple aggregate measurements over a 2-s integration time and standardized against a calibrated spectralon panel.

2015 PHT experiment foliar spectral collections. Spectral measurements were made on leaves from 62 cultivars with a total of 688 plants measured; opportunistic sampling resulted in differences in the number of plants assayed per cultivar. Foliar measurements at the PHT were made between 0900 and $1500 \mathrm{~h}$. Plants were first screened by trained seed certification inspectors in the field for symptoms of PVY; then, asymptomatic leaves from the identified plants were collected. Regardless of the presumed infection status of the plant, foliar sampling was standardized by selecting terminal leaflets from the penultimate whorl of leaves for spectral measurements not displaying symptoms; these leaves are furthermore referred to as "asymptomatic". To facilitate foliar collections from numerous plants in a row, these leaves were removed with scissors and spectral measurements were collected within 5 min of clipping. PVY status of leaves was determined using ImunoStrips for PVY (Agdia, Inc., Elkhart, IN) or by triple-antibody sandwich enzyme-linked immunosorbent assay (TAS-ELISA); leaves that did not test positive for PVY were classified as healthy in further statistical analyses.

The TAS-ELISA testing followed procedures described by Nikolaeva et al. (2012). Four monoclonal antibodies were used in this assay to determine the serotype of the PVY strain present: 4C3, 1F5, SASA-N, and MAb2. Monoclonal antibodies 4C3, 1F5 (Ellis et al. 1997), and MAb2 (McDonald and Kristjansson 1993) from were obtained from Agdia, Inc. or from Phyto Diagnostics (North Saanich, BC, Canada). The 4C3 antibody recognizes all PVY strains; 1F5 detects $\mathrm{PVY}^{\mathrm{N}}$, $\mathrm{PVY}^{\mathrm{NTN}}$, and $\mathrm{PVY}{ }^{\mathrm{O} 5}$; and $\mathrm{MAb} 2$ detects $\mathrm{PVY}^{\mathrm{O}}, \mathrm{PVY}^{\mathrm{N}-\mathrm{Wi}}$, and PVYC'. SASA-N was obtained from the Scottish Agricultural Science Agency (SASA, Edinburgh, Scotland) and is specific to $\mathrm{PVY}^{\mathrm{N}}$ and $\mathrm{PVY} \mathrm{Y}^{\mathrm{NTN}}$.

Samples identified by TAS-ELISA as positive were subjected to immunocapture reverse-transcription polymerase chain reaction (RTPCR) assays, as described by Lorenzen et al. (2006) and Chikh-Ali et al. (2013). The Lorenzen et al. (2006) assay can distinguish the nonrecombinant $\mathrm{PVY} \mathrm{Y}^{\mathrm{O}}$ and $\mathrm{PVY}^{\mathrm{N}}$ strains from recombinant $\mathrm{PVY} \mathrm{Y}^{\mathrm{NTN}}$ and $\mathrm{PVY}^{\mathrm{N}-\mathrm{Wi} / \mathrm{N}: \mathrm{O}}$ strains; however, no distinction can be made between $\mathrm{PVY}^{\mathrm{N}: \mathrm{O}}$ and $\mathrm{PVY} \mathrm{N}^{\mathrm{N}-\mathrm{Wi}}$ strains, and no distinction is possible between PVY ${ }^{\text {NA-N }}$ and PVY-NE11 strains. To separate these strains correctly, an additional multiplex RT-PCR assay was completed according to Chikh-Ali et al. (2013).

2014 HARS mechanical inoculation experiment foliar spectral collections. Foliar spectral measurements were made at HARS between 1100 and $1300 \mathrm{~h}$. Foliar sampling followed the leaf selection and spectral collection protocol used at the PHT, except spectral measurements were made on attached leaves. Prior to each spectral 
measurement, leaf adaxial temperature was measured with an infrared thermometer to account for any influence of temperature changes on spectroscopic retrievals. Foliar nitrogen, leaf mass per area (LMA), lignin, and maximum photosynthetic capacity $\left(\mathrm{V}_{\mathrm{cmax}}\right)$ were estimated from spectra using calibrations from Serbin (2012), which include model performance and stability metrics. Although potato gas exchange measurements were included in the model used for $\mathrm{V}_{\mathrm{cmax}}$, measurements from potato foliar samples were not included in models estimating nitrogen, LMA, and lignin. Nitrogen levels, however, were within a range of values found in comparative trials assessing nitrogen availability (Couture, Navaro, Bethke, and Townsend, unpublished data). Although it is possible that LMA and lignin values are not accurate in an absolute form, the relative variation and direction in the trait responses are likely accurate (Couture et al. 2015) and supported by generalized plant responses to pathogen infections (Nicholson and Hammerschmidt 1992). Chlorophyll content was determined from the relative difference between reflectance at wavelengths 750 and 705 (Gitelson and Merzlyak 1994); photochemical reflectance index (PRI, a measure of photosynthetic lightuse efficiency and plant stress) (Gamon et al. 1997) was calculated based on the relative difference between reflectance at wavelengths 531 and 570 (Gamon et al. 1997); and normalized differential water index
(NDWI, a measure of foliar water content) was determined from the relative difference in reflectance at wavelengths 857 and 1,241 (Gao 1996).

Models for nitrogen, lignin, LMA, and $\mathrm{V}_{\mathrm{cmax}}$ were originally built using a different spectrometer (FieldSpec 3; Analytical Spectral Design, Boulder, $\mathrm{CO}$ ) and, to make the models comparable across instruments, we transformed the SVC spectra into FS3 spectra using a wavelength-specific correction factor generated by making consecutive measurements on leaves $(n=20)$ with both instruments. After applying the correction factor, the spectral outputs were highly correlated across all wavelengths $(r=0.999)$.

Statistical approach. Differences in spectral profiles among leaves from infected and noninfected plants and different potato cultivars from the PHT were determined using permutational multivariate analysis of variance (PerMANOVA) (Anderson 2001) and visualized using principal components ordination. Classification of infection status and cultivar discrimination from samples from the PHT data set was performed using partial-least squares (PLS) discriminate analysis (DA). PLS-DA is an extension of the classical PLS regression (PLSR) algorithm, which allows robust model fitting in situations where issues of predictor multicollinearity preclude the use of classical least-squares approaches, or when the model is severely overspecified (i.e., more predictors than observations). The
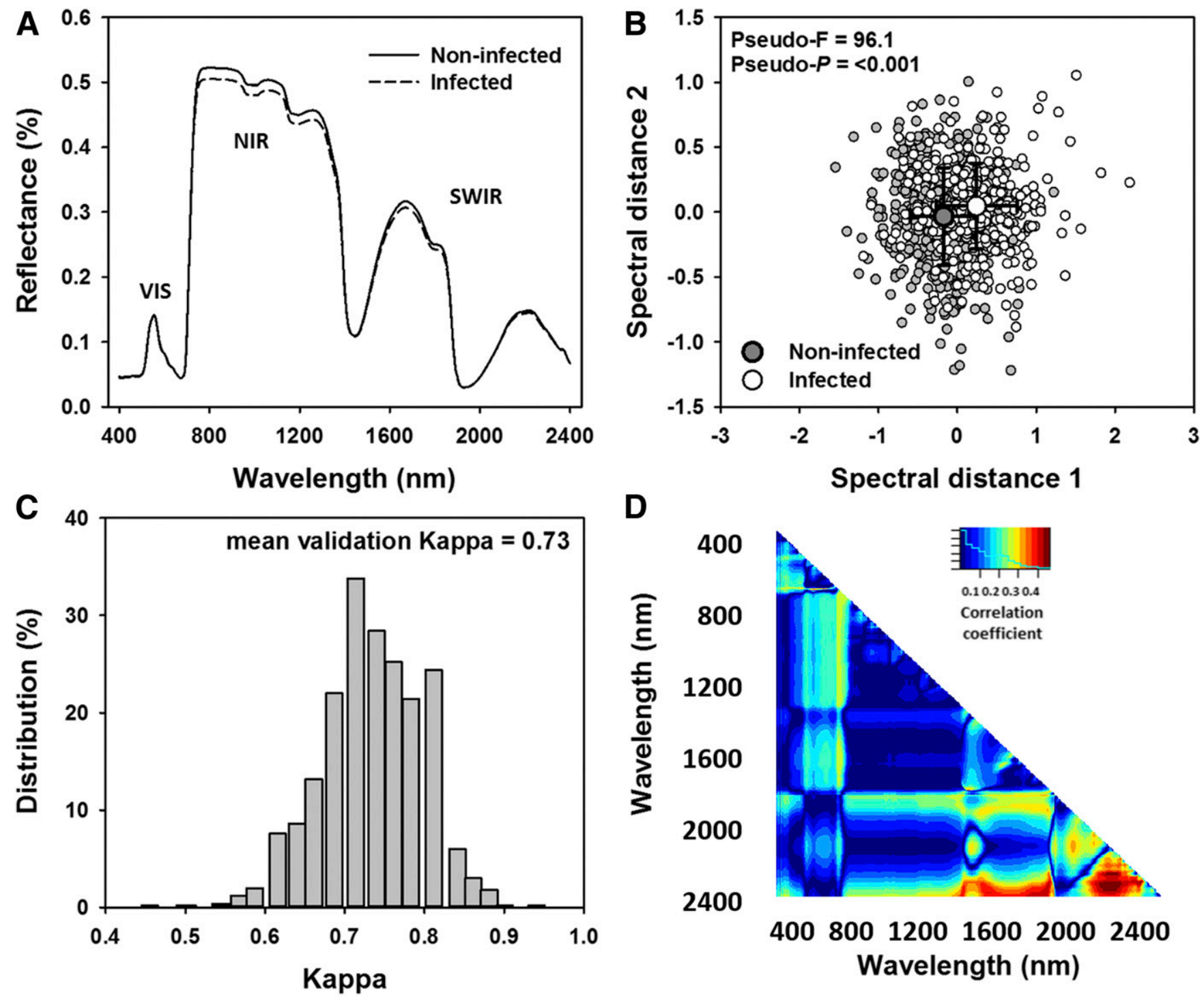

Fig. 1. A, Foliar reflectance profiles (VIS = visible region, NIR = near-infrared region, and SWIR = shortwave infrared region) of noninfected or Potato virus $Y(P V Y)$-infected leaves; B. ordination plot of the mean and standard error of first two axes depicting the dissimilarity of the composite spectral profiles of noninfected and PVY-infected leaves; C, distribution of the validation $\kappa$ statistic from the model classifying PVY infection over 500 permutations of the data set utilizing a 80/20 calibration/validation split; and D, normalized differential spectral index of all possible pairwise spectral combinations demonstrating relationships with plant infection status. 
PLS-DA algorithm is a retooling of the classic PLSR model that works by recoding response variables into dummy variables (i.e., one vector each for each class, with each occurrence of a class coded as ones and the rest as zeros) and determining coefficients for each vector that minimize the predicted variance in the response. Once the model has converged, the predicted vectors are recombined into putative probabilities using a softmax function that enforces a unity sum constraint. Model performance was evaluated using 500 randomized permutations of the dataset, with $80 \%$ of the data used for internal calibration and $20 \%$ for cross-validation. This approach generated a distribution of model performance across the multiple iterations allowing us to assess model stability and uncertainty. For each iteration, the model $\kappa$ statistic for the validation data set was tracked.

Spearman rank correlations between infection status and normalized differential spectral indexes [NDSI $=$ (wavelength $i-$ wavelength $j$ )/(wavelength $i+$ wavelength $j$ )] for all possible combinations of wavelengths were used to determine the strength of specific spectral regions for the classification of infection status. Differences among plant biochemical and biophysical responses to pathogen infection from HARS were determined using analysis of covariance following the model $Y_{j k l}=b_{j}+\mathrm{I}_{k}+\mathrm{V}_{l}+\mathrm{IV}_{k l}+t$. In this model, $b$ represents block $i$, I represents infection status $k$, V represents cultivar $l$, and $t$ represents the leaf temperature at the time of measurement. We included leaf temperature at the time of spectral measurements to account for any influence that temperature may have on the spectral retrievals. PerMANOVA, PLS-DA, and correlation analyses were conducted in R v. 3.2.1 (www.r-project.org) using the vegan, caret, and pls packages, while analysis of variance was conducted in JMP v. 12.0.1 (SAS Institute Inc., Cary, NC).

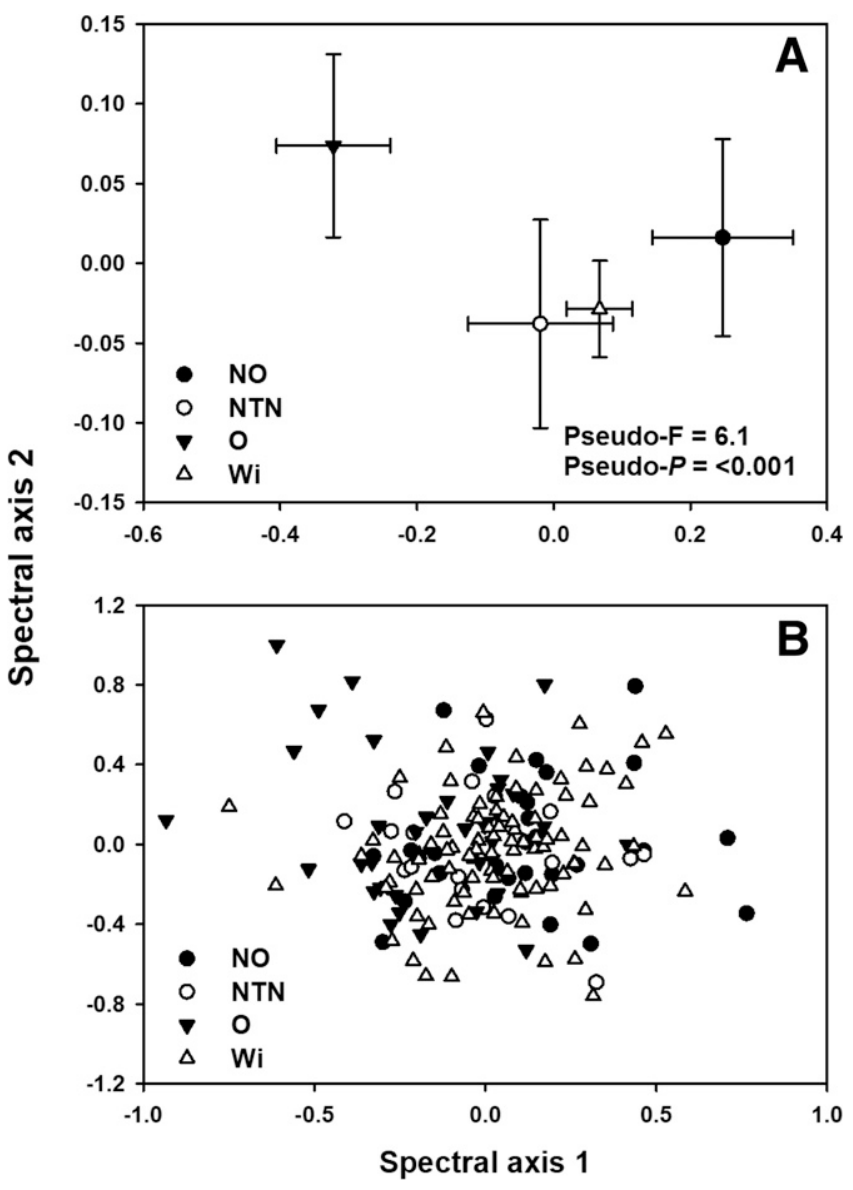

Fig. 2. A, Ordination plot of the mean and standard error of first two spectral axes depicting the dissimilarity of the composite spectral profiles of the four strains of Potato virus Y (PVY) (NO, NTN, O, and Wi) found in leaves of infected plants and B, ordination plot of the first two spectral axes of the composite spectral profiles of individual leaves of plants infected with one of the four PVY strains.

\section{Results}

Detection of PVY in asymptomatic leaves and determination of specific PVY strains from plants at the 2015 PHT experiment. Visual inspection of spectral profiles of healthy and asymptomatic infected leaves revealed spectral separation in the NIR and SWIR
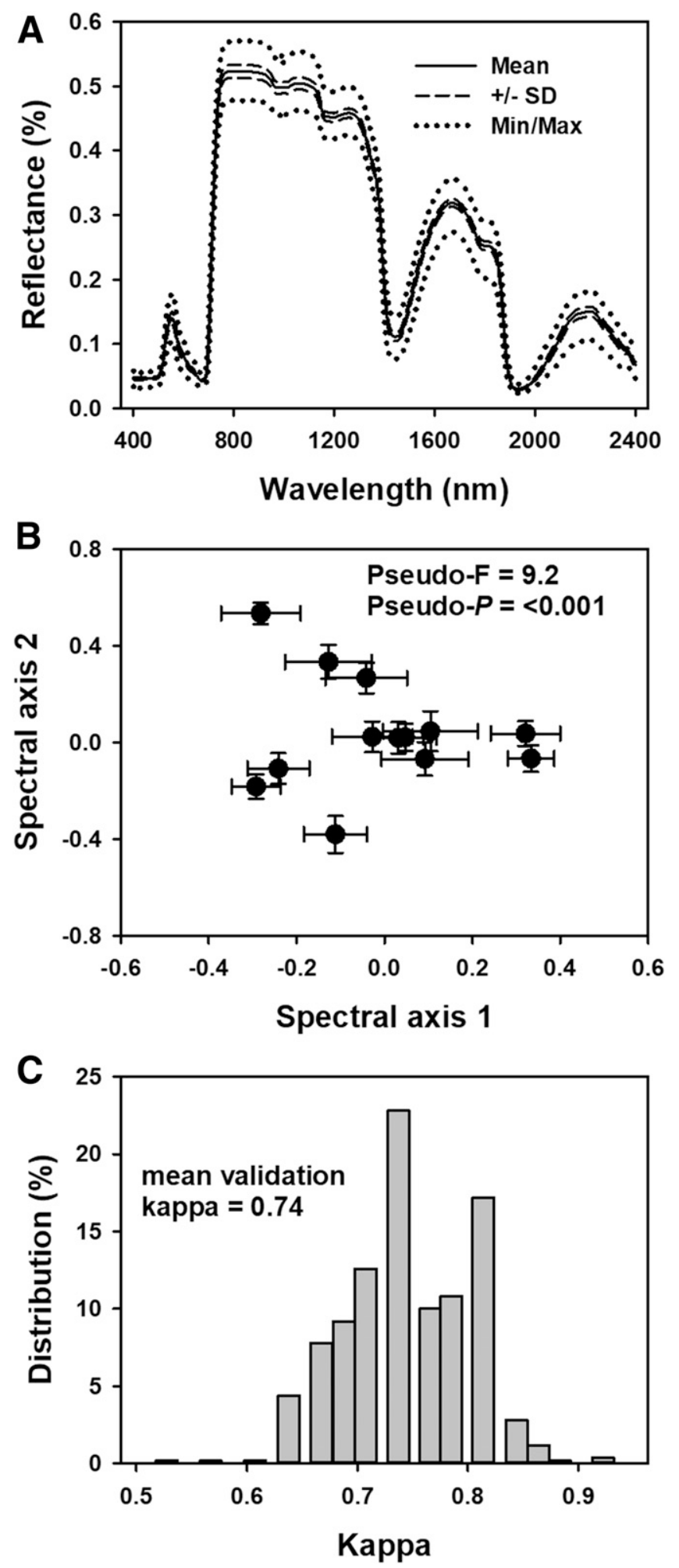

Fig. 3. A, Variation in foliar spectral profiles of leaves used for identification of potato cultivars ( $\mathrm{SD}=$ standard deviation); $\mathrm{B}$, ordination plot of the mean and standard error of first two axes depicting the dissimilarity of the composite spectral profiles of twelve cultivars of potato; and $\mathbf{C}$, distribution of the validation $\kappa$ statistic from the model classifying potato cultivars over 500 permutations of the data set utilizing a 80/20 calibration/validation split. 
spectral regions but nonsignificant differences in the visible region (Fig. 1A). Composite spectral profiles of PVY-negative and PVY-positive plants were significantly different (pseudo- $F=96.1$, pseudo- $P<0.001$ ) but considerable variation existed within each treatment (Fig. 1B). PLS-DA correctly characterized positive PVY infection status in vivo with a mean cross-validation $\kappa$ of 0.73 (range: 0.45 to 0.94 ) (Fig. 1C). NDSI in the narrowband regions within the SWIR showed the strongest relationships with infection status (Fig. 1D). Composite spectral profiles of specific PVY strains in infected leaves were statistically different (Fig. 2A) but substantial spectral variability existed among individual samples within each strain (Fig. 2B). The ability of spectral profiles to characterize different PVY strains was low, with a mean validation accuracy of approximately $12 \%$.

Identification of potato cultivars from plants at the 2015 PHT experiment. Spectral profiles showed considerable variation among different potato cultivars, predominately in the NIR and SWIR (Fig. 3A and B). In general, specific potato cultivars could be identified based on spectral profiles of validation data (validation $\kappa=0.74$ ) (Fig. 3C) but classification accuracies varied among cultivars (56 to 93\%) (Table 1). Prediction accuracies, however, were affected by pathogen presence and appeared to have a threshold minimum. Average classification accuracy of cultivars decreased when measurements of PVY-infected plants were included in the classification model (validation $\kappa=0.45$ ) and when cultivars with fewer than 10 healthy plants were included in the classification model (validation $\kappa=0.60$ ). After surpassing this threshold minimum, the number of samples included in the DA was not correlated with the classification accuracy $(r=0.20, P=0.51)$.

Plant physiological responses to PVY infection at the 2014 HARS mechanical inoculation experiment. PVY infection status and cultivar both influenced multiple potato foliar traits (Tables 2 and 3; Fig. 4). Leaf nitrogen concentrations and chlorophyll content were nominally affected by PVY infection but demonstrated variability among potato cultivars (Tables 2 and 3 ). $\mathrm{V}_{\mathrm{cmax}}$ declined approximately $15 \%$ in PVY-infected plants (Tables 2 and 3; Fig. 4) and the response was consistent across cultivars, with an overall $15 \%$ variation among potato cultivars (Tables 2). LMA and PRI increased approximately 55 and 53\% in PVY-infected plants, respectively; and, although the magnitude of the responses were variable, the direction of the responses were consistent across cultivars (Tables 2 and 3; Fig. 4). Leaf water content (reported as NDWI) was not influenced by PVY infection but varied approximately $25 \%$ across potato cultivars

Table 1. Summary of confusion matrix results indicating proportion of classification accuracies, and misclassifications, for individual potato cultivars with at least 10 measurements from healthy leaves ${ }^{\mathrm{a}}$

\begin{tabular}{|c|c|c|c|c|c|c|c|c|c|c|c|c|c|}
\hline Cultivar & ATL (31) & CRus (11) & DNR (24) & GOLD (19) & LAM (15) & NIC (16) & $\begin{array}{c}\text { PIKE } \\
\text { (19) }\end{array}$ & RN (13) & RusB (13) & RusN (12) & SIL (23) & SNO (28) & SUP (30) \\
\hline$\overline{\text { ATL }}$ & 0.90 & 0.00 & 0.01 & 0.00 & 0.00 & 0.00 & 0.03 & 0.00 & 0.00 & 0.00 & 0.03 & 0.02 & 0.00 \\
\hline CRus & 0.00 & 0.82 & 0.00 & 0.00 & 0.00 & 0.00 & 0.01 & 0.00 & 0.04 & 0.00 & 0.04 & 0.00 & 0.09 \\
\hline DNR & 0.02 & 0.00 & 0.81 & 0.01 & 0.01 & 0.04 & 0.00 & 0.00 & 0.00 & 0.00 & 0.02 & 0.01 & 0.07 \\
\hline GOLD & 0.00 & 0.00 & 0.04 & 0.93 & 0.00 & 0.00 & 0.00 & 0.00 & 0.00 & 0.00 & 0.02 & 0.00 & 0.00 \\
\hline LAM & 0.00 & 0.00 & 0.02 & 0.00 & 0.89 & 0.00 & 0.00 & 0.00 & 0.00 & 0.00 & 0.00 & 0.08 & 0.00 \\
\hline NIC & 0.02 & 0.00 & 0.10 & 0.02 & 0.00 & 0.78 & 0.00 & 0.00 & 0.00 & 0.00 & 0.03 & 0.03 & 0.00 \\
\hline PIKE & 0.06 & 0.00 & 0.03 & 0.00 & 0.00 & 0.00 & 0.77 & 0.00 & 0.00 & 0.00 & 0.00 & 0.07 & 0.04 \\
\hline RN & 0.02 & 0.00 & 0.03 & 0.10 & 0.00 & 0.03 & 0.00 & 0.67 & 0.00 & 0.00 & 0.05 & 0.10 & 0.00 \\
\hline RusB & 0.16 & 0.03 & 0.00 & 0.02 & 0.03 & 0.00 & 0.00 & 0.00 & 0.72 & 0.02 & 0.02 & 0.00 & 0.00 \\
\hline RusN & 0.05 & 0.00 & 0.01 & 0.05 & 0.02 & 0.00 & 0.07 & 0.00 & 0.06 & 0.69 & 0.03 & 0.02 & 0.00 \\
\hline SIL & 0.09 & 0.02 & 0.01 & 0.07 & 0.00 & 0.03 & 0.07 & 0.00 & 0.02 & 0.01 & 0.56 & 0.12 & 0.01 \\
\hline SNO & 0.04 & 0.00 & 0.02 & 0.00 & 0.07 & 0.01 & 0.00 & 0.08 & 0.00 & 0.00 & 0.14 & 0.64 & 0.00 \\
\hline SUP & 0.00 & 0.00 & 0.04 & 0.00 & 0.00 & 0.00 & 0.00 & 0.00 & 0.00 & 0.03 & 0.00 & 0.00 & 0.92 \\
\hline
\end{tabular}

a Each row represents the correct (bolded) and incorrect (nonbolded) classifications for a specific cultivar. Values are means of 500 permutations of partial least squares discriminate analysis. Potato cultivar abbreviations: $\mathrm{ATL}=$ Atlantic, $\mathrm{CRus}=\mathrm{Classic}$ Russet, DNR $=\mathrm{Dark}$ Red Norland, GOLD = Goldrush, LAM = Lamoka, NIC $=$ Nicolet, PIKE $=$ Pike, RN = Red Norland, RusB = Russet Burbank, RusN = Russet Norkotah, $\mathrm{SIL}=\mathrm{Silverton}, \mathrm{SNO}=\mathrm{Snowden}, \mathrm{SUP}=\mathrm{Su}-$ perior. Number below the cultivar abbreviation represents the number of independent leaf samples included in the discriminate analysis.

Table 2. Estimates of biochemical, physiological, and morphological characteristics retrieved from spectroscopic models for multiple cultivars of potato plants either infection free

\begin{tabular}{|c|c|c|c|c|c|c|c|c|c|c|c|c|c|c|}
\hline \multirow[b]{2}{*}{ Cultivar $^{b}$} & \multicolumn{2}{|c|}{ Nitrogen $(\% \mathrm{dm})$} & \multicolumn{2}{|c|}{ Chlorophyll $\left(\mathrm{g} \mathrm{m}^{-2}\right)$} & \multicolumn{2}{|c|}{$\mathrm{V}_{\mathrm{cmax}}\left(\mu \mathrm{mols} \mathrm{m}^{-2} \mathrm{~s}^{-1}\right)$} & \multicolumn{2}{|c|}{ LMA $\left(\mathrm{g} \mathrm{m}^{-2}\right)$} & \multicolumn{2}{|c|}{ PRI } & \multicolumn{2}{|c|}{ NDWI } & \multicolumn{2}{|c|}{ Lignin (\% dm) } \\
\hline & UTC & NTN & UTC & NTN & UTC & NTN & UTC & NTN & UTC & NTN & UTC & NTN & UTC & NTN \\
\hline ATL & $2.9 \pm 0.1$ & $2.8 \pm 0.1$ & $0.294 \pm 0.1$ & $0.268 \pm 0.1$ & $171.7 \pm 5.3$ & $150.0 \pm 5.2$ & $25.8 \pm 7.7$ & $47.1 \pm 7.6$ & $0.018 \pm 0.002$ & $0.030 \pm 0.002$ & $0.050 \pm 0.002$ & $0.055 \pm 0.002$ & $17.7 \pm 0.7$ & $21.1 \pm 0.7$ \\
\hline DRN & $2.7 \pm 0.1$ & $2.5 \pm 0.1$ & $0.320 \pm 0.1$ & $0.320 \pm 0.1$ & $172.6 \pm 7.2$ & $133.1 \pm 5.3$ & $33.4 \pm 10.4$ & $62.0 \pm 7.7$ & $0.022 \pm 0.003$ & $0.029 \pm 0.002$ & $0.064 \pm 0.003$ & $0.068 \pm 0.002$ & $21.7 \pm 1.0$ & $23.8 \pm 0.7$ \\
\hline RNK & $3.1 \pm 0.1$ & $3.2 \pm 0.1$ & $0.357 \pm 0.1$ & $0.357 \pm 0.1$ & $179.9 \pm 5.2$ & $155.4 \pm 5.7$ & $36.3 \pm 7.6$ & $53.4 \pm 8.3$ & $0.016 \pm 0.002$ & $0.021 \pm 0.003$ & $0.054 \pm 0.002$ & $0.052 \pm 0.003$ & $19.5 \pm 0.7$ & $20.7 \pm 0.8$ \\
\hline SUP & $3.1 \pm 0.1$ & $3.1 \pm 0.1$ & $0.329 \pm 0.1$ & $0.318 \pm 0.1$ & $172.2 \pm 5.6$ & $153.5 \pm 6.1$ & $41.7 \pm 8.1$ & $44.1 \pm 8.9$ & $0.015 \pm 0.003$ & $0.028 \pm 0.003$ & $0.053 \pm 0.003$ & $0.054 \pm 0.002$ & $20.7 \pm 0.8$ & $21.2 \pm 0.8$ \\
\hline
\end{tabular}

a Data represent mean responses \pm standard error. UTC $=$ untreated control and NTN $=$ infected $($ Potato virus $Y$ NTN $)$. $\mathrm{V}_{\mathrm{cmax}}=$ maximum rate of carboxylation,

LMA $=$ leaf mass per area, PRI $=$ photochemical reflectance index, NDWI $=$ normalized differential water index, and $\%$ dm $=\%$ dry mass.

${ }^{\mathrm{b}} \mathrm{ATL}=$ Atlantic, DRN = Dark Red Norland, RNK = Russet Norkotah, and SUP = Superior.

Table 3. Summary of $F$ and $P$ values of the effects of infection status, cultivar, and their interaction on potato foliar chemistry, morphology, and physiology as estimated by reflectance spectroscopy ${ }^{\mathrm{a}}$

\begin{tabular}{|c|c|c|c|c|c|c|c|c|c|c|c|c|c|c|c|}
\hline \multirow[b]{2}{*}{ Treatments } & \multirow[b]{2}{*}{ df } & \multicolumn{2}{|c|}{ Nitrogen } & \multicolumn{2}{|c|}{ Chlorophyll } & \multicolumn{2}{|c|}{$V_{\text {cmax }}$} & \multicolumn{2}{|c|}{ LMA } & \multicolumn{2}{|c|}{ PRI } & \multicolumn{2}{|c|}{ NDWI } & \multicolumn{2}{|c|}{ Lignin } \\
\hline & & $F$ & $P$ & $F$ & $P$ & $F$ & $P$ & $F$ & $P$ & $F$ & $P$ & $F$ & $P$ & $F$ & $P$ \\
\hline Infection & 1 & 0.9 & 0.344 & 1.0 & 0.312 & 49.2 & $<0.001$ & 9.3 & 0.002 & 28.7 & $<0.001$ & 1.9 & 0.163 & 12.4 & $<0.001$ \\
\hline Cultivar & 3 & 7.1 & $<0.001$ & 10.4 & $<0.001$ & 1.5 & 0.222 & 5.4 & 0.438 & 1.7 & 0.171 & 15.5 & $<0.001$ & 8.2 & $<0.001$ \\
\hline Infection $\times$ cultivar & 3 & 0.7 & 0.545 & 0.5 & 0.687 & 1.6 & 0.191 & 0.4 & 0.310 & 1.1 & 0.339 & 1.4 & 0.235 & 1.7 & 0.165 \\
\hline Temperature & 1 & 2.3 & 0.131 & 7.1 & 0.010 & 1.8 & 0.178 & 3.9 & 0.057 & 1.6 & 0.213 & 0.2 & 0.677 & 4.3 & 0.042 \\
\hline
\end{tabular}

a $P$ values $<0.05$ are in bold. $\mathrm{LMA}=$ leaf mass per area, NDWI $=$ normalized differential water index, $\mathrm{PRI}=$ photochemical reflectance index, $\mathrm{V}_{\mathrm{cmax}}=$ maximum rate of carboxylation, and $\mathrm{df}=$ degrees of freedom. 
(Tables 2 and 3). Foliar lignin concentrations varied by approximately $23 \%$ across cultivars (Table 2 ) and increased approximately 9\% with PVY infection (Table 2; Fig. 4); although the magnitude of the increase in lignin in response to PVY was variable, it was consistent across cultivars (Tables 2 and 3; Fig. 4).

\section{Discussion}

Here, we demonstrate that hyperspectral data can be used to (i) detect PVY infection in asymptomatic leaves; (ii) identify specific potato cultivars; and (iii) estimate specific chemical, morphological, and physiological responses of potato plants to PVY infection. This demonstrates that information obtained from hyperspectral data can significantly advance precision agricultural approaches, potentially increasing management efficiency within cropping systems. Although logistical challenges exist, the acquisition of full-range hyperspectral data, and its applications, can be extended to larger spatial scales via unmanned aerial vehicles and other airborne platforms, facilitating the implementation of spectroscopy as a quantitative
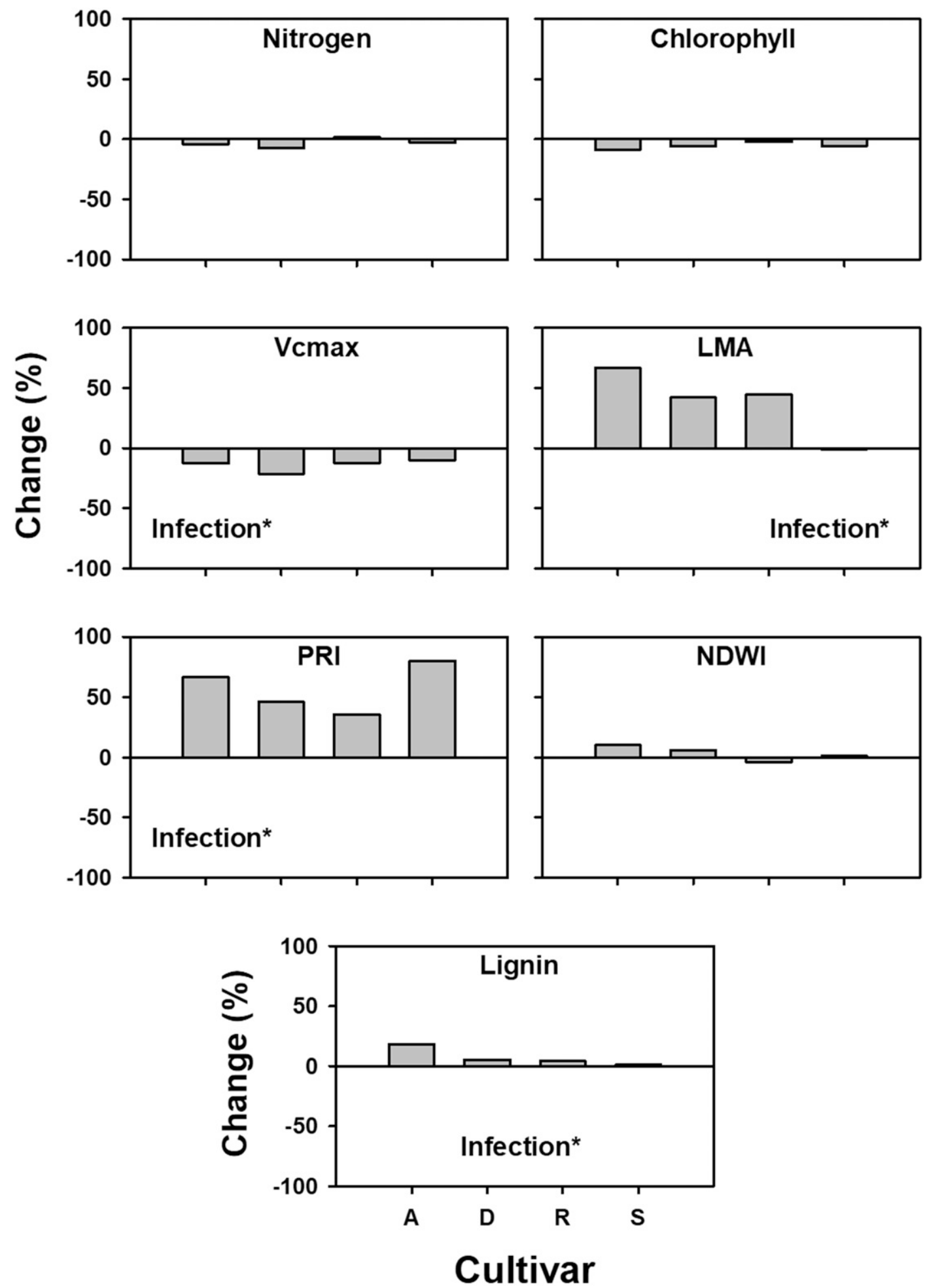

Fig. 4. Mean relative foliar biochemical, morphological, and physiological responses of multiple cultivars of potato plants to Potato virus $Y$ (PVY) infection. Percent change was determined as [(uninfected response - infected response)/uninfected response] $\times 100 . V_{c m a x}=$ maximum rate of carboxylation, $L M A=l e a f$ mass per area, $P R I=$ photochemical reflectance index, and NDWI = normalized differential water index. Cultivars: $A=$ Atlantic, $D=$ Dark Red Norland, $R=$ Russet Norkotah, and $S=$ Superior. An asterisk indicates statistically significant treatment effect at $P<0.05$. 
measurement tool for precision agriculture and optimized management practices. It is worth noting, however, that spectral features enabling disease detection at the leaf level may differ at the canopy level (Herrmann et al. 2018).

Pathogen infection is a major cause of economic loss in most cropping systems, including potato. Hyperspectral data have been shown to effectively detect pathogen presence in other systems (Arens et al. 2016; Delalieux et al. 2007; Mahlein et al. 2012, 2013; Naidu et al. 2009; Rumpf et al. 2010). Many of these studies, however, made spectral measurements on foliage that was already visually expressing disease symptoms, including necrosis, chlorosis, and shifts in leaf pigments. Here, we demonstrated the ability of spectral information to detect PVY infection in asymptomatic leaves. Moreover, we assessed this ability across a range of cultivar and virus combinations, which can generate a range of visual responses (Gray et al. 2010). This outcome suggests that this approach does not rely on highly specific plant cultivar-PVY strain interactions for virus detection and, therefore, our findings suggest the potential for management actions before visual symptoms appear. Quicker management responses such as removal of virus-infected plants allow for treating smaller, localized infection points, potentially limiting pathogen spread.

Although different strains of PVY produced different foliar spectral profiles, the ability to identify individual PVY strains from spectral data was poor. Different PVY strains produce different foliar symptoms, and these symptoms vary with host cultivar, plant age, and plant nutrition. Although there are differences in gene expression of potato to different PVY isolates (Kogovšek et al. 2010), the early molecular, biochemical, and physiological responses to infection by different PVY strains are poorly characterized. Similarities in responses of plants to different PVY strains before symptoms are expressed may limit the ability of spectral data to discriminate between different PVY strains at early stages of infection. Importantly, our assessment of the ability of spectroscopy to identify PVY presence in asymptomatic leaves is constrained to a single developmental stage (i.e., preflowering), thus limiting our understanding of the sensitivity of this approach to disease detection over the course of the infection process. Future work relating early-stage, asymptomatic molecular, biochemical, and physiological profiles to foliar spectral data over the course of the infection process will be needed to resolve relationships among plant optical properties and strain-specific infection status over the infection process.

We found that hyperspectral data successfully identified the different potato cultivars examined, although the classification accuracies depended on PVY infection status and the number of samples included in the model, and varied among cultivars. Our results are similar to those of Arens et al. (2016), who were able to discriminate among four cultivars of sugar beet, ranging in susceptibility to a fungal pathogen, using hyperspectral data. In addition, similar to our findings, the authors identified regions of the SWIR as being the most variable between susceptible and resistant cultivars. Studies examining the ability of hyperspectral information to classify cultivars generally do so on uninfected plants (Arens et al. 2016), potentially missing the impact of pathogen infection of cultivar discrimination. Here, we show that infection substantially decreases cultivar classification accuracy, a conclusion similar to that of Rajah et al. (2015), who reported differential classification accuracies of different dry bean (Phaseolus vulgaris L.) cultivars based on length of exposure to water stress. The decrease in cultivar classification accuracy as a result of PVY presence suggests that the impact of PVY on the foliar optical properties of potato may potentially mask cultivar-specific traits that enable spectral determination of different cultivars The influence of PVY on relationships among foliar optical properties and cultivar-specific traits, however, requires further research to better understand the capability of spectral data to classify individual cultivars in the presence or absence of different stress events. We also show that, when PVY-free, some cultivars are more accurately characterized than are others, a likely emergent property of the overlap in the spectral profiles among the cultivars used in our analysis and a consequence of the traits for which different cultivars have been bred. Although utilizing spectral information for cultivar classification is rapidly emerging, plant infection, stress status, and phenological stage should be considered when interpreting classification results, and research exploring multistress experiments and implementing classification approaches that filter responses to multiple biotic and abiotic variables will be needed to accurately categorize crop status. This is especially true in field settings, where growing conditions can be highly variable, especially when extending hyperspectral approaches to larger spatial scales using airborne platforms.

A powerful, yet underutilized, approach in precision agriculture is the ability of hyperspectral information to estimate specific plant biochemical and physiological properties. We found that PVY infection affected leaf chemical and physiological responses, and a number of the responses we report are consistent with previous findings related to pathogen-infected plants. We found a modest increase in leaf lignin content, a physical defense response often associated with pathogen infection (Nicholson and Hammerschmidt 1992; Xu et al. 2011). Although pathogen infection can also influence plant water relations (Grimmer et al. 2012), we found no evidence that pathogen infection affected leaf water content. We also found no influence of infection status on chlorophyll content, a finding similar to that of Zhou et al. (2004), who reported that substantial reductions in chlorophyll content did not occur until 60 days postinfection. Our findings of decreased photosynthetic capacity agree with those of others who found reductions in photosynthetic activity in potato infected with PVY ${ }^{\mathrm{NTN}}$ (Stare et al. 2015; Zhou et al. 2004). In addition, PRI, a spectroscopically derived index of photosynthetic efficiency that putatively tracks xanthophyll cycling (Gamon et al. 1997), was positively related with $\mathrm{V}_{\mathrm{cmax}}(r=0.42, P=<0.001)$. The similarities of our findings produced using spectroscopy with those from standard analytical techniques suggest that hyperspectral information has the potential to provide a great deal of information about detailed physiological responses of plants to pathogen infection.

In conclusion, we found that hyperspectral information can successfully detect pathogen infection status prior to the onset of visual symptoms and is able to discriminate between specific plant cultivars, with the latter depending on disease status. In addition, a novel application of hyperspectral data we present is the quantification of specific physiological responses of potato plants to PVY infection. The application and integration of information from multiple scientific disciplines with sensor and monitoring technologies will help advance the rapidly growing area of precision agriculture. In particular, this approach lends itself well to field-based sampling of potato for early disease detection, quantification of specific physiological responses, and assessing cultivar purity.

\section{Literature Cited}

Anderson, M. J. 2001. A new method for non-parametric multivariate analysis of variance. Austral Ecol. 26:32-46.

Arens, N., Backhaus, A., Dŏll, S., Fischer, S., Seiffert, U., and Mock, H.-P. 2016 Detection of Cercospora beticola infection and identification of early metabolic responses in sugar beet. Front. Plant Sci. 7:1377.

Asner, G. P., and Martin, R. E. 2008. Spectral and chemical analysis of tropical forests: Scaling from leaf to canopy levels. Remote Sens. Environ. 112:3958-3970.

Asner, G. P., Martin, R. E., Tupayachi, R., Emerson, R., Marinez, P., Sinca, F., Powell, G. V. N., Wright, S. J., and Lugo, A. E. 2011. Taxonomy and remote sensing of leaf mass per area (LMA) in humid tropical forests. Ecol. Appl. 21:85-98.

Chikh-Ali, M., Gray, S. M., and Karasev, A. V. 2013. An improved multiplex ICRT-PCR assay distinguishes nine strains of Potato virus Y. Plant Dis. 97: 1370-1374.

Coombs, J. J., Frank, L. M., and Douches, D. S. 2004. An applied fingerprinting system for cultivated potato using simple sequence repeats. Am. J. Potato Res. 81:243-250.

Cotrozzi, L., Couture, J. J., Cavender-Bares, J., Kingdon, C. C., Fallon, B., Pilz, G. Pellegrini, E., Nali, C., and Townsend, P. A. 2017. Using foliar spectral properties to assess the effects of drought on plant water potential. Tree Physiol. 37:1582-1591.

Couture, J. J., Serbin, S. P., and Townsend, P. A. 2013. Spectroscopic sensitivity of real-time, rapidly induced phytochemical change in response to damage. New Phytol. 198:311-319.

Couture, J. J., Serbin, S. P., and Townsend, P. A. 2015. Elevated temperature and periodic water stress alter growth and quality of common milkweed (Asclepias 
syriaca) and monarch (Danaus plexippus) larval performance. Arthropod-Plant Interact. 9:149-161.

Couture, J. J., Singh, A., Rubert-Nason, K. F., Serbin, S. P., Lindroth, R. L., and Townsend, P. A. 2016. Spectroscopic determination of ecologically relevant plant secondary metabolites. Methods Ecol. Evol. 7:1402-1412.

Curran, P. J. 1989. Remote sensing of foliar chemistry. Remote Sens. Environ. 30: 271-278.

Delalieux, S., van Aardt, J., Keulemans, W., Schrevens, E., and Coppin, P. 2007. Detection of biotic stress (Venturia inaequalis) in apple trees using hyperspectral data: Non-parametric statistical approaches and physiological implications. Eur. J. Agron. 27:130-143.

Di Carli, M., Benvenuto, E., and Donini, M. 2012. Recent insights into plant-virus interactions through proteomic analysis. J. Proteome Res. 11:4765-4780.

Douches, D. S., and Ludlam, K. 1991. Electrophoretic characterization of North American potato cultivars. Am. Potato J. 68:767-780.

Ellis, P., Stace-Smith, R., and de Villers, G. 1997. Identification and geographic distribution of serotypes of potato virus Y. Plant Dis. 81:481-484.

Frost, K. E., Groves, R. L., and Charkowski, A. O. 2013. Integrated control of potato pathogens through seed potato certification and provision of clean seed potatoes. Plant Dis. 97:1268-1280.

Gamon, J. A., Field, C. B., Goulden, M. L., Griffin, K. L., Hartley, A. E., Joel, G., Peñuelas, J., and Valentini, R. 1995. Relationship between NDVI, canopy structure, and photosynthesis in three Californian vegetation types. Ecol. Appl. 5:28-41.

Gamon, J. A., Peñuelas, J., and Field, C. B. 1992. A narrow-waveband spectral index that tracks diurnal changes in photosynthetic efficiency. Remote Sens. Environ. 41:35-44

Gamon, J. A., Serrano, L., and Surfus, J. S. 1997. The photochemical reflectance index: An optical indicator of photosynthetic radiation use efficiency across species, functional types, and nutrient levels. Oecologia 112:492-501.

Gao, B. 1996. NDWI-A normalized difference water index for remote sensing of vegetation liquid water from space. Remote Sens. Environ. 58:257-266.

Gitelson, A. A., and Merzlyak, M. N. 1994. Spectral reflectance changes associate with autumn senescence of Aesculus hippocastanum L. and Acer platanoides L. leaves. Spectral features and relation to chlorophyll estimation. J. Plant Physiol. 143:286-292.

Gray, S., De Boer, S., Lorenzen, J., Karasev, A., Whitworth, J., Nolte, P., Singh, R., Boucher, A., and Xu, H. 2010. Potato virus $Y$ : An evolving concern for potato crops in the United States and Canada. Plant Dis. 94:1384-1397.

Grimmer, M. K., Foulkes, M. J., and Paveley, N. D. 2012. Foliar pathogenesis and plant water relations: A review. J. Exp. Bot. 63:4321-4331.

Guenthner, J. F., Johnson, A. J., and McIntosh, C. S. 2012. Seed variety mix: An indicator for GM potato identity preservation. Am. J. Potato Res. 89: 172-174.

Herrmann, I., Vosberg, S. K., Ravindran, P., Singh, A., Chang, H.-X., Chilvers, M. I., Conley, S. P., and Townsend, P. A. 2018. Leaf and canopy level detection of Fusarium virguliforme (sudden death syndrome) in soybean. Remote Sens. 10:426.

Huang, W., Lamb, D. W., Niu, Z., Zhang, Y., Liu, L., and Wang, J. 2007. Identification of yellow rust in wheat using in-situ spectral reflectance measurements and airborne hyperspectral imaging. Precision Agric. 8:187-197.

Kleinebecker, T., Schmidt, S. R., Fritz, C., Smolders, A. J. P., and Holzel, N. 2009. Prediction of delta ${ }^{13} \mathrm{C}$ and delta ${ }^{15} \mathrm{~N}$ in plant tissues with near-infrared reflectance spectroscopy. New Phytol. 184:732-739.

Kogovšek, P., Pompe-Novak, M., Baebler, Š., Rotter, A., Gow, L., Gruden, K., Foster, G. D., Boonham, N., and Ravnikar, M. 2010. Aggressive and mild Potato virus $Y$ isolates trigger different specific responses in susceptible potato plants. Plant Pathol. 59:1121-1132

Li, H., He, Y., and Fang, H. 2007. Non-destructive discrimination of Chinese bayberry varieties using Vis/NIR spectroscopy. J. Food Eng. 81:357-363.

$\mathrm{Li}, \mathrm{X}$., and He, Y. 2008. Discriminating varieties of tea plant based on Vis/NIR spectral characteristics and using artificial neural networks. Biosyst. Eng. 99: 313-321.
Lorenzen, J. H., Piche, L. M., Gudmestad, N. C., Meacham, T., and Shiel, P. 2006. A multiplex PCR assay to characterize Potato virus $Y$ isolates and identify strain mixtures. Plant Dis. 90:935-940.

Mahlein, A.-K., Rumpf, T., Welke, P., Dehne, H.-W., Plümer, L., Steiner, U., and Oerke, E.-C. 2013. Development of spectral indices for detecting and identifying plant diseases. Remote Sens. Environ. 128:21-30.

Mahlein, A.-K., Steiner, U., Hillnhütter, C., Dehne, H.-W., and Oerke, E.-C. 2012 Hyperspectral imaging for small-scale analysis of symptoms caused by different sugar beet diseases. Plant Methods 8:3.

Martinelli, F., Scalenghe, R., Davino, S., Panno, S., Scuderi, G., Rusis, P., Villa, P., Stroppiana, D., Boschetti, M., Goulart, L. R., Davis, C. E., and Dandekar, A. M 2015. Advanced methods of plant disease detection. A review. Agron. Sustain. Dev. 35:1-25

McDonald, J. G., and Kristjansson, G. T. 1993. New diseases and epidemics properties of strains of Potato virus $\mathrm{Y}^{\mathrm{N}}$ in North America. Plant Dis. 77: 87-89.

Naidu, R. A., Perry, E. M., Pierce, F. J., and Mekuria, T. 2009. The potential of spectral reflectance technique for the detection of Grapevine leafroll-associated virus-3 in two red-berried wine grape cultivars. Comput. Electron. Agric. 66:38-45.

Nicholson, R. L., and Hammerschmidt, R. 1992. Phenolic compounds and their role in disease resistance. Annu. Rev. Phytopathol. 30:369-389.

Nikolaeva, O. V., Roop, D. J., Galvino-Costa, B. F., dos Reis Figueira, A., Gray, S. M. and Karasev, A. V. 2012. Epitope mapping for monoclonal antibodies recognizing tuber necrotic isolates of Potato virus Y. Am J. Potato Res. 89:121-128.

Petisco, C., Garcia-Criado, B., Mediavilla, S., de Aldana, B. R. V., Zabalgogeazcoa, I., and Garcia-Ciudad, A. 2006. Near-infrared reflectance spectroscopy as a fast and non-destructive tool to predict foliar organic constituents of several woody species. Anal. Bioanal. Chem. 386:1823-1833.

Rajah, P., Odindi, J., Abdel-Rahman, E. M., Mutanga, O., and Modi, A. 2015. Varietal discrimination of common dry bean (Phaseolus vulgaris L.) grown under different watering regimes using multitemporal hyperspectral data. J. Appl. Remote Sens. 9:096050.

Romero, A. P., Alarcón, A., Valbuena, R. I., and Galeano, C. H. 2017 Physiological assessment of water stress in potato using spectral information. Front. Plant Sci. 8:1608.

Rumpf, T., Mahlein, A.-K., Steiner, U., Oecke, E.-C., Dehne, H.-W., and Plümer, L. 2010. Early detection and classification of plant diseases with support vector machines based on hyperspectral reflectance. Comput. Electron. Agric. 74: 91-99.

Sankaran, S., Mishra, A., Ehsani, R., and Davis, C. 2010. A review of advanced techniques for detecting plant diseases. Comput. Electron. Agric. 72:1-13.

Serbin, S. P. 2012. Spectroscopic determination of leaf nutritional, morphological, and metabolic traits. Ph.D. dissertation, University of Wisconsin-Madison, Madison

Serbin, S. P., Dillaway, D. N., Kruger, E. L., and Townsend, P. A. 2012. Leaf optical properties reflect variation in photosynthetic metabolism and its sensitivity to temperature. J. Exp. Bot. 63:489-502.

Serbin, S. P., Singh, A., McNeil, B. E., Kingdon, C. C., and Townsend, P. A. 2014 Spectroscopic determination of leaf morphological and biochemical traits for northern temperate and boreal tree species. Ecol. Appl. 24:1651-1669.

Stare, T., Ramšak, Ž., Blejec, A., Stare, K., Turnšek, N., Weckwerth, W. Wienkoop, S., Vodnik, D., and Gruden, K. 2015. Bimodal dynamics of primary metabolism related responses in tolerant potato-Potato virus $Y$ interaction. BMC Genomics 16:716.

Xu, L., Zhu, L., Tu, L., Liu, T., Yuan, D., Jin, L., Long, L., and Zhang, X. 2011. Lignin metabolism has a central role in the resistance of cotton to the wilt fungus Verticillium dahliae as revealed by RNA-Seq-dependent transcriptional analysis and histochemistry. J. Exp. Bot. 62:5607-5621.

Zhou, Y. H., Peng, Y. H., Lei, J. L., Zou, L. Y., Zheng, J. H., and Yu, J. Q 2004. Effects of Potato virus $\mathrm{Y}^{\text {NTN }}$ infection on gas exchange and photosystem 2 function in leaves of Solanum tuberosum L. Photosynthetica 42:417-423. 